\title{
CONTINUOUS HAHN POLYNOMIALS OF DIFFERENTIAL OPERATOR ARGUMENT AND ANALYSIS ON RIEMANNIAN SYMMETRIC SPACES OF CONSTANT CURVATURE
}

\author{
ERICH BADERTSCHER AND TOM H. KOORNWINDER
}

\begin{abstract}
For the three types of simply connected Riemannian spaces of constant curvature it is shown that the associated spherical functions can be obtained from the corresponding (zonal) spherical functions by application of a differential operator of the form $p(i d / d t)$, where $p$ belongs to a system of orthogonal polynomials: Gegenbauer polynomials, Hahn polynomials or continuous symmetric Hahn polynomials. We give a group theoretic explanation of this phenomenon and relate the properties of the polynomials $p$ to the properties of the corresponding representation. The method is extended to the case of intertwining functions.
\end{abstract}

1. Introduction. This paper deals with the remarkable phenomenon that associated spherical functions on Euclidean space and on the spaces of constant positive and negative curvature can be obtained from the corresponding spherical functions by application of a differential operator which can be written as $p_{k}(i d / d t)$, where the $p_{k}$ form some explicit system of orthogonal polynomials.

The most simple situation of this phenomenon can be found on $\mathbb{R}^{2}$, where the Bessel function $J_{0}$ is a spherical function and the Bessel functions $J_{k}\left(k \in \mathbb{Z}_{+}\right)$are associated spherical functions. The $J_{k}$ fulfill the following differential recurrence relation:

$$
2 J_{k}^{\prime}(t)=J_{k-1}(t)-J_{k+1}(t)
$$

On the other hand the three term recurrence relation for the Chebyshev polynomials $T_{k}$ $\left(k \in \mathbb{Z}_{+}\right)$of the first kind is given by

$$
2 x T_{k}(x)=T_{k-1}(x)+T_{k+1}(x) .
$$

By comparing the two formulas we obtain by induction on $k$

$$
T_{k}\left(-i \frac{d}{d t}\right) J_{0}(t)=i^{k} J_{k}(t)
$$

(for $k=0,1$ the formula is directly verified).

Received by the editors August 21, 1990 .

AMS subject classification: 43A90, 43A85, 33C80, 33C45.

Key words and phrases: spaces of constant curvature, spherical functions, associated spherical functions, Bessel functions, Jacobi functions, Gegenbauer polynomials, Hahn polynomials, continuous symmetric Hahn polynomials.

(c) Canadian Mathematical Society 1992. 
A much more sophisticated but non-explicit theorem of Helgason [13] points into the same direction. Let $G$ be a connected semisimple Lie group with finite center. Let $K$ be a maximal compact subgroup of $G$. Helgason [13, Theorem 4.1] proves that, in the generic case, any joint eigenfunction of the $G$-invariant differential operators on $G / K$ which belongs to an irreducible $K$-type can be obtained from the corresponding spherical function by application of some (on $G$ ) right-invariant differential operator.

In our relatively simple setting of Euclidean spaces, spheres and hyperboloids we will obtain explicit analogues of (1.1) by group theoretic methods. The polynomials $p_{k}$ in terms of which the differential operator is expressed will be Gegenbauer polynonials, Hahn polynomials and continuous symmetric Hahn polynomials, respectively. Closely associated with this result are formulas giving the explicit classical Fourier transform of the spherical function $t \longmapsto \phi_{\lambda}(t)$ (already derived in Section 2). For the noncompact case this formula looks quite remarkable.

Here are the contents of the paper. In Section 2 of the paper we introduce the various special functions we shall need in the paper and we derive the just mentioned Fourier transforms.

In Section 3 we give an analytic derivation of the generalization of formula (1.1) to Bessel functions of arbitrary index $\alpha \in \mathbb{C}$. This formula is obtained by specializing an even more general formula for Bessel functions and Gegenbauer polynomials of a certain partial differential operator.

In Section 4 we give the group theoretic interpretation of the curious formulas including special functions and orthogonal polynomials of differential operators. The orthogonal polynomials $p_{k}$ associated by us to a spherical representation $\pi$ are also strongly connected with the intrinsic structure of the representation. In particular $\pi$ is degenerate if and only if the family $p_{k}$ is degenerate and $\pi$ is unitarizable if and only if the family $p_{k}$ is positive definite.

In Section 5 we show that the moment functionals describing the polynomials $p_{k}$ come from weight functions on $\mathbb{R}$ that are nothing but the ordinary Fourier transforms of the spherical functions corresponding to $\pi$. Since we know these transforms explicitly, we can now also identify the polynomials $p_{k}$ explicitly as Gegenbauer polynomials, as Hahn polynomials, as continous Hahn polynomials respectively.

In Section 6 we show how the polynomials $p_{k}$ can be used to obtain the associated spherical functions associated to $\pi$ from the spherical function of $\pi$. Since we know all involved functions explicitly, this leads to our various generalizations of formula (1.3).

In Section 7 we use the fact, that our method is not restricted to spherical functions associated to $\pi$. It applies to arbitrary $K$-left-invariant generalized matrix elements of $\pi$. We illustrate the method by choosing two particular families of intertwining functions on homogeneous spaces of $G$.

Many formulas of this paper have been announced in [18, Sections 2-4]. 
2. Preliminaries on special functions. Here we introduce the various special functions that will be encountered in the paper. We use the familiar ${ }_{p} F_{q}$ notation in the definitions.

Jacobi functions:

$$
\phi_{\lambda}^{(\alpha, \beta)}(t):={ }_{2} F_{1}\left(\frac{1}{2}(\alpha+\beta+1+i \lambda), \frac{1}{2}(\alpha+\beta+1-i \lambda) ; \alpha+1 ;-\sinh ^{2} t\right),
$$

$c f$. for instance [17]. For $\operatorname{Re} \alpha>-\frac{1}{2}$ the Jacobi function $\phi_{\lambda}^{\left(\alpha,-\frac{1}{2}\right)}$ has the integral representation

$$
\phi_{\lambda}^{\left(\alpha,-\frac{1}{2}\right)}(t)=\frac{\Gamma(\alpha+1)}{\Gamma\left(\alpha+\frac{1}{2}\right) \Gamma\left(\frac{1}{2}\right)} \int_{0}^{\pi}(\cosh t+\sinh t \cos \psi)^{i \lambda-\alpha-\frac{1}{2}}(\sin \psi)^{2 \alpha} d \psi
$$

Formula (2.2) is a degenerate case of the integral representation $[17,(5.28)]$ for Jacobi functions, but can also be derived from an integral representation $[6,3.7(8)]$ for associated Legendre functions by substitution of

$$
\phi_{\lambda}^{\left(\alpha,-\frac{1}{2}\right)}(t)=2^{\alpha} \Gamma(\alpha+1)(\sinh t)^{-\alpha} P_{-\frac{1}{2}+i \lambda}^{-\alpha}(\cosh t),
$$

(cf. $[6,3.2(20)])$. From formula (2.2) we can obtain explicitly the Fourier transform of $\phi_{\lambda}^{\left(\alpha,-\frac{1}{2}\right)}$; substitute $\cos \psi=-\tanh y$ in the integral on the right hand side in order to transform it into a convolution:

$$
\phi_{\lambda}^{\left(\alpha,-\frac{1}{2}\right)}(t)=\frac{\Gamma(\alpha+1)}{\Gamma\left(\alpha+\frac{1}{2}\right) \Gamma\left(\frac{1}{2}\right)} \int_{-\infty}^{\infty}(\cosh (t-y))^{i \lambda-\alpha-\frac{1}{2}}(\cosh y)^{-i \lambda-\alpha-\frac{1}{2}} d y .
$$

For $\operatorname{Im} \lambda<\operatorname{Re} \alpha+\frac{1}{2}$, the function $y \longmapsto(\cosh y)^{-i \lambda-\alpha-\frac{1}{2}}$ belongs to the class $S$ of rapidly decreasing $C^{\infty}$-functions on $\mathbb{R}$ with explicit Fourier transform given by

$\int_{-\infty}^{\infty}(\cosh y)^{-i \lambda-\alpha-\frac{1}{2}} e^{i \mu y} d y=\frac{2^{i \lambda+\alpha-\frac{1}{2}}}{\Gamma\left(i \lambda+\alpha+\frac{1}{2}\right)} \Gamma\left(\frac{1}{2}\left(i \mu+i \lambda+\alpha+\frac{1}{2}\right)\right) \Gamma\left(\frac{1}{2}\left(-i \mu+i \lambda+\alpha+\frac{1}{2}\right)\right)$

(cf. $[6,1.5(26)])$. We can apply (2.4) to the convolution (2.3) in order to obtain the Fourier transform of $t \longmapsto \phi_{\lambda}^{\left(\alpha,-\frac{1}{2}\right)}(t)$ for $|\operatorname{Im} \lambda|<\operatorname{Re} \alpha+\frac{1}{2}$ :

$$
\int_{-\infty}^{\infty} \phi_{\lambda}^{\left(\alpha,-\frac{1}{2}\right)}(t) e^{i \mu t} d t=\frac{2^{2 \alpha-1} \Gamma(\alpha+1) w_{\alpha, \lambda}(\mu)}{\Gamma\left(\alpha+\frac{1}{2}\right) \Gamma\left(\frac{1}{2}\right) \Gamma\left(i \lambda+\alpha+\frac{1}{2}\right) \Gamma\left(-i \lambda+\alpha+\frac{1}{2}\right)},
$$

where $w_{\alpha, \lambda}(\mu)$ is given by

$$
\begin{aligned}
w_{\alpha, \lambda}(\mu)=\Gamma\left(\frac{1}{2}\left(i \mu+i \lambda+\alpha+\frac{1}{2}\right)\right) \Gamma\left(\frac{1}{2}\left(-i \mu+i \lambda+\alpha+\frac{1}{2}\right)\right) \\
\\
\quad \times \Gamma\left(\frac{1}{2}\left(i \mu-i \lambda+\alpha+\frac{1}{2}\right)\right) \Gamma\left(\frac{1}{2}\left(-i \mu-i \lambda+\alpha+\frac{1}{2}\right)\right) .
\end{aligned}
$$

By Fourier inversion we have for $|\operatorname{Im} \lambda|<\operatorname{Re} \alpha+\frac{1}{2}$ :

$$
\phi_{\lambda}^{\left(\alpha,-\frac{1}{2}\right)}(t)=\frac{2^{2 \alpha-2} \Gamma(\alpha+1)}{\pi^{3 / 2} \Gamma\left(\alpha+\frac{1}{2}\right) \Gamma\left(i \lambda+\alpha+\frac{1}{2}\right) \Gamma\left(-i \lambda+\alpha+\frac{1}{2}\right)} \int_{-\infty}^{\infty} w_{\alpha, \lambda}(\mu) e^{-i \mu t} d \mu .
$$

This formula will play an essential role in Section 5. 
Jacobi polynomials:

$$
R_{k}^{(\alpha, 3)}(x):=\frac{k !}{(\alpha+1)_{k}} P_{k}^{(\alpha, 3)}(x)={ }_{2} F_{1}\left(-k, k+\alpha+\beta+1 ; \alpha+1 ; \frac{1}{2}(1-x)\right),
$$

where $P_{k}^{\left(\alpha,{ }^{3}\right)}$ is the usual Jacobi polynomial. For $\alpha, \beta>-1$ these are orthogonal polynomials on the interval $(-1,1)$ with respect to the weight function $(1-x)^{\alpha}(1+x)^{3}$. See Szegö [22, Chapter 4]. For $\alpha=\beta$, Szegö $[22,(4.9 .19),(4.9 .21),(4.7 .6)]$ gives the explicit Fourier series

$$
R_{k}^{(\alpha, \alpha)}(\cos \theta)=\sum_{j=0}^{k} w_{k-2 j}^{\alpha, k} e^{i(2 j-k) \theta}
$$

with

$$
w_{k-2 j}^{\alpha, k}:=\frac{\left(\alpha+\frac{1}{2}\right)_{k-j}\left(\alpha+\frac{1}{2}\right)_{j} k !}{(k-j) ! j !(2 \alpha+1)_{k}} .
$$

Bessel functions:

$$
j_{\alpha}(z):=\Gamma(\alpha+1)\left(\frac{1}{2} z\right)^{-\alpha} J_{\alpha}(z)={ }_{0} F_{1}\left(\alpha+1 ;-z^{2} / 4\right),
$$

where $J_{\alpha}$ is the usual Bessel function. There is an integral representation

$$
j_{\alpha}(t)=\frac{\Gamma(\alpha+1)}{\Gamma\left(\alpha+\frac{1}{2}\right) \Gamma\left(\frac{1}{2}\right)} \int_{-1}^{1} e^{-i t x}\left(1-x^{2}\right)^{\alpha-\frac{1}{2}} d x, \quad \operatorname{Re} \alpha>-\frac{1}{2},
$$

cf. $[25,3.3(4)]$.

Continuous symmetric Hahn polynomials:

$$
p_{k}(x ; a, b):=i^{k}{ }_{3} F_{2}\left(\begin{array}{c}
-k, k+2 a+2 b-1, a-i x \\
a+b, 2 a
\end{array} ;\right) .
$$

These are orthogonal polynomials on $\mathbb{R}$ with respect to the weight function $x \mapsto$ $|\Gamma(a+i x) \Gamma(b+i x)|^{2}(a, b>0$ or $b=\bar{a}$, Re $a>0)$. See Askey and Wilson [1], [2, p.48]. By (2.5) the monic orthogonal polynomials $p_{k}$ on $\mathbb{R}$ with respect to the weight function $w_{\alpha, \lambda}\left(|\operatorname{Im} \lambda|<\alpha+\frac{1}{2}\right)$ are given in terms of continuous symmetric Hahn polynomials as

$$
p_{k}(\mu):=\frac{(-1)^{k}(2 \alpha)_{k}\left(\frac{1}{2}+\alpha+i \lambda\right)_{k}}{2^{k}(\alpha)_{k}} p_{k}\left(\frac{\mu}{2} ; \frac{1}{4}+\frac{\alpha}{2}+\frac{i \lambda}{2}, \frac{1}{4}+\frac{\alpha}{2}-\frac{i \lambda}{2}\right) .
$$

Note that $p_{k}(\mu)$ is an even function of $\lambda$.

Hahn polynomials:

$$
Q_{k}(x ; \beta, \gamma, N):={ }_{3} F_{2}\left(\begin{array}{c}
-k, k+\beta+\gamma+1,-x \\
\beta+1,-N
\end{array}\right), \quad k=0,1, \ldots, N .
$$

These are orthogonal polynomials on $\{0,1, \ldots, N\}$ with respect to the weights

$$
x \mapsto\left(\begin{array}{c}
\beta+x \\
x
\end{array}\right)\left(\begin{array}{c}
N-x+\gamma \\
N-x
\end{array}\right)
$$


cf. Karlin and McGregor [15], Askey and Wilson [2, p.47]. By (2.8), (2.9) the monic orthogonal polynomials $p_{k}$ on $\{-n,-n+2, \ldots, n-2, n\}$ with respect to the weights $\mu \longmapsto w_{\mu}^{\alpha, n}$ are given in terms of Hahn polynomials as

$$
p_{k}(\mu)=\frac{(-1)^{k}(2 \alpha)_{k}(-n)_{k}}{2^{k}(\alpha)_{k}} Q_{k}\left(\frac{1}{2}(n-\mu) ; \alpha-\frac{1}{2}, \alpha-\frac{1}{2}, n\right) .
$$

3. The Euclidean case. Let $d=2,3, \ldots$ Let $S^{d-1}$ be the unit sphere in the Euclidean space $\mathbb{R}^{d}$ with normalized surface measure $d \sigma$. For $x, y \in \mathbb{R}^{d}$ let $x . y$ denote their inner product and $|x|$ the length of $x$. Then, for each homogeneous harmonic polynomial $Y$ of degree $k$ on $\mathbb{R}^{d}$ we have

$$
\int_{S^{d-1}} e^{i \xi-x} Y(\xi) d \sigma(\xi)=\frac{\left(\frac{1}{2} i\right)^{k}}{\left(\frac{1}{2} d\right)_{k}} j_{\frac{1}{2} d-1+k}(|x|) Y(x), \quad x \in \mathbb{R}^{d},
$$

where $j_{\alpha}$ is the Bessel function in modified notation defined by (2.10). See for instance Faraut [10, Corollary II.8]. In particular, for $k=0$ this becomes

$$
\int_{S^{d-1}} e^{i \xi x} d \sigma(\xi)=j_{\frac{1}{2} d-1}(|x|), \quad x \in \mathbb{R}^{d} .
$$

PROPOSITION 3.1. Let Y be a homogeneous harmonic polynomial of degree $k$ on $\mathbb{R}^{d}$. Then

$$
Y\left(-i \partial / \partial x_{1}, \ldots,-i \partial / \partial x_{d}\right) j_{\frac{1}{2} d-1}(|x|)=\frac{\left(\frac{1}{2} i\right)^{k}}{\left(\frac{1}{2} d\right)_{k}} j_{\frac{1}{2} d-1+k}(|x|) Y(x), \quad x \in \mathbb{R}^{d}
$$

PROOF. Apply the differential operator $Y\left(-i \partial / \partial x_{1}, \ldots,-i \partial / \partial x_{d}\right)$ to both sides of (3.2) and compare with (3.1).

The special case $d=3$ of (3.3) goes back to Van der Pol [20] and Erdélyi [5], see also $[7,11.5(32)]$. We thank A. Strasburger for these references.

In particular, if we take

$$
Y\left(x_{1}, \ldots, x_{d}\right):=\sum_{j=0}^{\left[\frac{1}{2} k\right]} c_{j} x_{1}^{k-2 j}|x|^{2 j}, \quad x \in \mathbb{R}^{d},
$$

where the $c_{j}$ are given by

$$
R_{k}^{\left(\frac{1}{2}(d-3), \frac{1}{2}(d-3)\right)}(x)=\sum_{j=0}^{\left[\frac{1}{2} k\right]} c_{j} x^{k-2 j}, \quad x \in \mathbb{R},
$$

(i.e., $Y$ is a zonal spherical harmonic), and if we use that

$$
\left(\frac{\partial^{2}}{\partial x_{1}^{2}}+\cdots+\frac{\partial^{2}}{\partial x_{d}^{2}}+1\right) j_{\frac{1}{2} d-1}(|x|)=0
$$


then we obtain from (3.3) that, for $x \in \mathbb{R}^{d}$,

$$
R_{k}^{\left(\frac{1}{2}(d-3), \frac{1}{2}(d-3)\right)}\left(-i \partial / \partial x_{1}\right) j_{\frac{1}{2} d-1}(|x|)=\frac{\left(\frac{1}{2} i\right)^{k}}{\left(\frac{1}{2} d\right)_{k}} j_{\frac{1}{2} d-1+k}(|x|)|x|^{k} R_{k}^{\left(\frac{1}{2}(d-3), \frac{1}{2}(d-3)\right)}\left(x_{1} /|x|\right) .
$$

In (3.4) put $x=(t \cos \theta, t \sin \theta, 0, \ldots, 0)$. Then we obtain the case $\alpha=\frac{1}{2} d-1$ of the next proposition.

PROPOSITION 3.2. For arbitrary complex $\alpha \neq-1 / 2,-1,-3 / 2, \ldots$ we have

$$
R_{k}^{\left(\alpha-\frac{1}{2}, \alpha-\frac{1}{2}\right)}\left(-i\left(\cos \theta \frac{\partial}{\partial t}-t^{-1} \sin \theta \frac{\partial}{\partial \theta}\right)\right) j_{\alpha}(t)=\frac{i^{k}}{2^{k}(\alpha+1)_{k}} R_{k}^{\left(\alpha-\frac{1}{2}, \alpha-\frac{1}{2}\right)}(\cos \theta) t^{k} j_{\alpha+k}(t) .
$$

In particular, for $\theta=0$,

$$
R_{k}^{\left(\alpha-\frac{1}{2}, \alpha-\frac{1}{2}\right)}(-i d / d t) j_{\alpha}(t)=\frac{i^{k}}{2^{k}(\alpha+1)_{k}} t^{k} j_{\alpha+k}(t)
$$

Proof. By rewriting [7, 7.8(12)], which is an integrated version of $[25,11.5(9)]$, we obtain that, for $\operatorname{Re} \alpha>-\frac{1}{2}$,

$$
\begin{aligned}
\frac{i^{k}}{2^{k}(\alpha+1)_{k}} R_{k}^{\left(\alpha-\frac{1}{2}, \alpha-\frac{1}{2}\right)}(\cos \theta) t^{k} j_{\alpha+k}(t) & \\
= & \frac{\Gamma(\alpha+1)}{\Gamma\left(\alpha+\frac{1}{2}\right) \Gamma\left(\frac{1}{2}\right)} \int_{0}^{\pi} e^{i t \cos \theta \cos \psi} \\
& \quad \times j_{\alpha-\frac{1}{2}}(t \sin \theta \sin \psi) R_{k}^{\left(\alpha-\frac{1}{2}, \alpha-\frac{1}{2}\right)}(\cos \psi)(\sin \psi)^{2 \alpha} d \psi
\end{aligned}
$$

and, in particular for $k=0$,

$$
j_{\alpha}(t)=\frac{\Gamma(\alpha+1)}{\Gamma\left(\alpha+\frac{1}{2}\right) \Gamma\left(\frac{1}{2}\right)} \int_{0}^{\pi} e^{i t \cos \theta \cos \psi} j_{\alpha-\frac{1}{2}}(t \sin \theta \sin \psi)(\sin \psi)^{2 \alpha} d \psi
$$

Now application of $R_{k}^{\left(\alpha-\frac{1}{2}, \alpha-\frac{1}{2}\right)}\left(-i\left(\cos \theta \partial / \partial t-t^{-1} \sin \theta \partial / \partial \theta\right)\right)$ to (3.8) sends its left hand side to the left hand side of (3.5) and its right hand side to the right hand side of (3.7). Thus we get (3.5) first for $\operatorname{Re} \alpha>-\frac{1}{2}$ and next, in general, by analytic continuation with respect to $\alpha$.

4. Orthogonal polynomials and the $K$-decomposition of a spherical representation. Let, for $d \geq 2, G$ be one of the groups $O_{0}(1, d), O(d+1), I\left(\mathbb{R}^{d}\right)$, and let $K$ be the subgroup $O(d)$. (For $d \geq 3$ we may also take $G:=\mathrm{SO}_{0}(1, d), \mathrm{SO}(d+1), I_{0}\left(\mathbb{R}^{d}\right)$, and $K:=\mathrm{SO}(d)$.) Let $M$ be the subgroup $O(d-1)$ of $K$. Both $(G, K)$ and $(K, M)$ are Gelfand pairs and $K / M$ can be identified with the unit sphere in $\mathbb{R}^{d}$. We will refer to the three cases for $G$ as the noncompact case, the compact case and the Euclidean case, respectively. See for instance Faraut $[9, \S \mathrm{II}]$ for generalities about these pairs $(G, K)$. 
Let $g$ and $f$ be the Lie algebras of $G$ and $K$, respectively. There is an involution of $g$ for which $f$ is the 1-eigenspace. Let $\mathfrak{p}$ be the $(-1)$-eigenspace. (In the Euclidean case, $\mathfrak{p}$ is just the Lie algebra of the translation group.) The group $K$ acts on $\mathfrak{p}$ via the adjoint representation. If $\mathfrak{g}=\mathfrak{f}+\mathfrak{p}$ is the Lie algebra of $G$ in the compact case then the Lie algebras of $G$ in the noncompact and Euclidean case can be identified with $f+i p$ (as real form of complexified $g$ ) and $f+p$ (as semidirect product), respectively. In the compact case, take an $\operatorname{Ad}(G)$-invariant inner product on $g$ such that $G / K$ has sectional curvature 1 (this is uniquely determined). By the just-mentioned correspondences between the Lie algebras, this inner product can be transferred to $\operatorname{Ad}(K)$-invariant inner products on $f$ and $\mathfrak{p}$ in the noncompact and Euclidean cases. In the noncompact case, the sectional curvature of $G / K$ resulting from the inner product on $\mathfrak{p}$ will be equal to -1 . Choose an orthonormal basis $X_{1}, \ldots, X_{d}$ of $\mathfrak{p}$ such that $X_{1}$ is $M$-fixed. Put $a:=\mathbb{R} X_{1}, A:=\exp a$.

Let $\pi$ be an irreducible $K$-finite $K$-unitary representation of $G$ on a Hilbert space $\mathcal{H}$ which is spherical, i.e., for which $\mathcal{H}$ has a normalized $K$-fixed vector $e_{0}$ (necessarily unique up to a phase factor, since $(G, K)$ is a Gelfand pair). Write $\langle.$, . $\rangle$ for the inner product on $\mathcal{H}$. Let $\mu$ be the moment functional which associates with any polynomial $p$ in one variable the number

write

$$
\mu(p):=\left\langle\pi\left(p\left(i X_{1}\right)\right) e_{0}, e_{0}\right\rangle,
$$

for the corresponding moments and write

$$
\langle p, q\rangle_{\mu}:=\left\langle\pi\left(p\left(i X_{1}\right) q\left(i X_{1}\right)\right) e_{0}, e_{0}\right\rangle
$$

for the associated bilinear form. Let $s$ be the biggest number in $\mathbb{N} \cup\{\infty\}$ such that $\langle.$, . $\rangle$ is non-degenerate on any space of polynomials of degree $k<s$ (or, equivalently, such that for $\left.k<s \operatorname{det}\left(\mu_{i+j}\right)_{i, j=0, \ldots, k} \neq 0\right)$. For $0 \leq k<s$ let $p_{k}$ be the monic orthogonal polynomial with respect to the moment functional $\mu$. (See Chihara [3, Chapter 1] for the general theory of orthogonal polynomials with respect to a moment functional.) Let

$$
e_{k}:=\pi\left(p_{k}\left(i X_{1}\right)\right) e_{0}, \quad 0 \leq k<s .
$$

We now state the two main theorems of this section.

THEOREM 4.1. For $0 \leq k<s$ the vector $e_{k}$ is $M$-invariant, nonzero and a cyclic vector for an irreducible $K$-module $\mathcal{H}^{k}$ which is $K$-equivariant to the space of spherical harmonics of degree $k$ on $K / M$. Furthermore,

$$
\mathcal{H}=\bigoplus_{0 \leq k<s} \mathcal{H}^{k}
$$

Finally, for any $k$ with $s \leq k<\infty$ the bilinearform $\langle.,$.$\rangle is degenerate on the space of$ polynomials of degree $\leq k$. 
THEOREM 4.2. The representation $\pi$ is unitarizable iff the bilinear form $\langle., .\rangle_{\mu}$ is positive definite on the space of polynomials of degree $<s$, i.e., iff

$$
\sum_{p, q \leq k} c_{p} \overline{c_{q}} \mu_{p+q}>0 \text { for } k<s \text { and } 0 \neq\left(c_{1}, \ldots, c_{k}\right) \in \mathbb{C}^{k} .
$$

We will prove these results in a sequence of lemmas and propositions. Here the polynomials $p_{k}$, the vectors $e_{k}$, the spaces $\mathcal{H}^{k}$ and the numbers $s$ will be defined first in a way different from above, but later it will turn out that for each object the two definitions agree.

We first study the action of the (complex) universal enveloping algebra $\mathcal{U}(\mathrm{g})$ on $e_{0}$. The natural filtration $\left\{\mathcal{U}_{k}(\mathrm{~g})\right\}_{k=0,1,2, \ldots}$ of $\mathcal{U}(\mathrm{g})$ induces a natural grading of $\mathcal{H}$ as follows. Put

$$
\mathcal{H}_{k}:=\pi\left(\mathcal{U}_{k}(g)\right) e_{0}
$$

and let $\mathcal{H}^{k}$ be the orthogonal complement of $\mathcal{H}_{k-1}$ in $\mathcal{H}_{k}$. Note that $\mathcal{H}=\oplus_{k=0}^{\infty} \mathcal{H}^{k}$ by irreducibility of $\pi$.

Let $S(\mathrm{~g})$ denote the symmetric algebra over the vector space $\mathrm{g}$ and let $S^{k}(\mathrm{~g})$ denote the space of elements in $S(g)$ which are homogeneous of degree $k$. Let $H^{k}(\mathfrak{p})$ be the subspace of $S^{k}(\mathfrak{p})$ for which the elements are harmonic polynomials on the linear dual $\mathfrak{p}^{*}$ of $\mathfrak{p}$. Let $\sigma: S(\mathrm{~g}) \rightarrow \mathcal{U}(\mathrm{g})$ denote the symmetrization mapping (cf. Helgason [14, Theorem II.4.3], where the mapping is denoted by $\lambda$ ).

LEMMA 4.3. Either the space $\mathcal{H}^{k}$ is $K$-irreducible or $\mathcal{H}^{k}=\{0\}$. The mapping $Y \longmapsto \pi(\sigma(Y)) e_{0}: S(\mathfrak{p}) \rightarrow \mathcal{H}$ intertwines the representations $\operatorname{Ad}$ and $\left.\pi\right|_{K}$ of $K$. We have

$$
\mathcal{H}^{k}=\pi\left(\sigma\left(H^{k}(\mathfrak{p})\right)\right) e_{0} .
$$

ProOF. Since

$$
\mathcal{U}_{k}(\mathrm{~g})=\mathcal{U}_{k-1}(\mathrm{~g})^{\mathfrak{f}}+\sigma\left(\sum_{i=0}^{k} S^{i}(\mathfrak{p})\right)
$$

(see Helgason [14, Lemma II.4.7]), we have

$$
\mathcal{H}_{k} \subset \pi\left(\sigma\left(S^{k}(\mathfrak{p})\right)\right) e_{0}+\mathcal{H}_{k-1}
$$

Next we know

$$
S^{k}(\mathfrak{p})=H^{k}(\mathfrak{p})+S^{k-2}(\mathfrak{p})\left(X_{1}^{2}+\cdots+X_{d}^{2}\right)
$$

(see e.g. Vilenkin [23, Chapter 9]) whence

$$
\sigma\left(S^{k}(\mathfrak{p})\right) \subset \sigma\left(H^{k}(\mathfrak{p})\right)+\sigma\left(S^{k-2}(\mathfrak{p})\right)\left(X_{1}^{2}+\cdots+X_{d}^{2}\right)+\mathcal{U}_{k-1}(\mathfrak{g}) .
$$

But

$$
\pi(k) \pi(\sigma(Y)) e_{0}=\pi(k) \pi(\sigma(Y)) \pi\left(k^{-1}\right) e_{0}=\pi(\sigma(\operatorname{Ad}(k) Y)) e_{0}, \quad Y \in S(\mathfrak{p}), k \in K .
$$


Hence the mapping $Y \mapsto \pi(\sigma(Y)) e_{0}: S(\mathfrak{p}) \rightarrow \mathcal{H}$ intertwines the representations Ad and $\left.\pi\right|_{K}$ of $K$. So, since $\mathrm{Ce}_{0}$ is the space of $K$-fixed elements in $\mathcal{H}, e_{0}$ is an eigenvector of $\pi\left(X_{1}^{2}+\cdots+X_{d}^{2}\right)$. Hence we obtain from (4.4) and (4.5) that

$$
\mathcal{H}_{k} \subset \pi\left(\sigma\left(H^{k}(\mathfrak{p})\right)\right) e_{0}+\mathcal{H}_{k-1}
$$

and, by iteration,

$$
\mathcal{H}_{k} \subset \sum_{i=0}^{k} \pi\left(\sigma\left(H^{i}(\mathfrak{p})\right)\right) e_{0}
$$

Clearly the inverse inclusion is also valid, so

$$
\mathcal{H}_{k}=\sum_{i=0}^{k} \pi\left(\sigma\left(H^{i}(\mathfrak{p})\right)\right) e_{0}
$$

But the spaces $H^{k}(\mathfrak{p})$ are $K$-irreducible and pairwise $K$-inequivalent (see for instance [23, Chapter 9]), so the statements of the lemma follow now from (4.6) and the Schur lemma.

Let $k \in \mathbb{Z}_{+}$and let $P_{k}$ be the polynomial in $n$ variables such that $P_{k}\left(X_{1}, \ldots, X_{n}\right)$ is the monic zonal (i.e. $M$-invariant) polynomial in $H^{k}(\mathfrak{p})$. Its coefficients can be obtained from a Gegenbauer polynomial power series expansion: Let

$$
R_{k}^{\left(\frac{1}{2}(d-3), \frac{1}{2}(d-3)\right)}(x)=c\left(x^{k}+a_{k-2} x^{k-2}+\cdots\right),
$$

then

$$
P_{k}\left(x_{1}, \ldots, x_{d}\right)=x_{1}^{k}+a_{k-2} x_{1}^{k-2}\left(x_{1}^{2}+\cdots+x_{d}^{2}\right)+\cdots .
$$

Define

$$
e_{k}:=\pi\left(\sigma\left(P_{k}\left(i X_{1}, \ldots, i X_{d}\right)\right)\right) e_{0}, \quad k \in \mathbb{Z}_{+}
$$

Then $e_{k}$ is an $M$-invariant vector in $\mathcal{H}^{k}$, unique up to a constant factor, and $e_{k}=0$ iff $\mathcal{H}^{k}=\{0\}$.

LEMMA 4.4. For each $k \in \mathbb{Z}_{+}$there is a monic polynomial $p_{k}$ in one variable of degree $k$ such that

$$
e_{k}=\pi\left(p_{k}\left(i X_{1}\right)\right) e_{0}
$$

PROOF. By elementary properties of the symmetrization mapping we have

$$
\begin{aligned}
e_{k} & =\pi\left(\sigma\left(\left(i X_{1}\right)^{k}+a_{k-2} i^{k-2} X_{1}^{k-2}\left(X_{1}^{2}+\cdots+X_{d}^{2}\right)+\cdots\right)\right) e_{0} \\
& \in \pi\left(\left(i X_{1}\right)^{k}\right) e_{0}+\mathcal{U}_{k-2}(\mathrm{~g})\left(X_{1}^{2}+\cdots+X_{d}^{2}\right) e_{0}+\mathcal{U}_{k-1}(\mathrm{~g}) e_{0} \\
& \subset \pi\left(\left(i X_{1}\right)^{k}\right) e_{0}+\mathcal{U}_{k-1}(\mathrm{~g}) e_{0} \\
& =\pi\left(\left(i X_{1}\right)^{k}\right) e_{0}+\sum_{i=0}^{k-1} H^{i} .
\end{aligned}
$$

As $e_{k}-\pi\left(\left(i X_{1}\right)^{k}\right) e_{0}$ is $M$-invariant, the result follows by complete induction with respect to $k$. 
PROPOSITION 4.5. There is a unique $\in \in \mathbb{N} \cup\{\infty\}$ such that dim $\mathcal{H}^{k}>0$ iff $k<s$. For this $s$ the vectors $e_{k}(k<s)$ are obtained by orthogonalization of the sequence $e_{0}$, $\pi\left(i X_{1}\right) e_{0}, \pi\left(\left(i X_{1}\right)^{2}\right) e_{0}, \ldots$ and, if $k<s$ or $k=s<\infty$, then the polynomials $p_{k}$ are the unique monic polynomials of degree $k$ satisfying (4.8). If $k=s<\infty$ then $\pi\left(p_{s}\left(i X_{1}\right)\right) e_{0}=$ 0 .

Proof. Choose $s \in \mathbb{N} \cup\{\infty\}$ maximal such that the vectors $\pi\left(\left(i X_{1}\right)^{k}\right) e_{0}(k<s)$ are linearly independent. Then, by (4.8), the vectors $e_{k}(k<s)$ are linearly independent as well and the statements about orthogonalization and uniqueness of the $p_{k}$ are evident. Our choice of $s$ clearly has the property required in the Proposition if $s=\infty$. Next, if $s<\infty$ then, by induction,

$$
\pi\left(\left(i X_{1}\right)^{k}\right) e_{0} \in \operatorname{span}\left\{e_{0}, \ldots, \pi\left(\left(i X_{1}\right)^{s-1}\right) e_{0}\right\} \subset \mathcal{H}_{s-1} \text { for all } k .
$$

Hence, for $g=k_{1} a k_{2} \in G\left(k_{1}, k_{2} \in K, a \in A\right)$ :

$$
\pi(g) e_{0}=\pi\left(k_{1}\right) \pi(a) e_{0} \in \pi\left(k_{1}\right) \mathcal{H}_{s-1} \subset \mathcal{H}_{s-1},
$$

whence, by irreducibility,

$$
\bigoplus_{k=0}^{\infty} \mathcal{H}^{k}=\mathcal{H} \subset \mathcal{H}_{s-1}=\bigoplus_{k=0}^{s-1} \mathcal{H}^{k}
$$

This shows that $\mathcal{H}^{k}=\{0\}$ if $k \geq s$, while we already know that $\mathcal{H}^{k} \neq\{0\}$ if $k<s$.

Let $\tilde{\pi}$ be the conjugate contragredient representation to $\pi$, i.e. the representation of $G$ on $\mathcal{H}$ such that

$$
\langle\pi(g) e, f\rangle=\left\langle e, \tilde{\pi}\left(g^{-1}\right) f\right\rangle, \quad e, f \in \mathcal{H}, g \in G .
$$

Then $\tilde{\pi}$ is also an irreducible $K$-finite $K$-unitary representation of $G$ on $\mathcal{H}$ and $\pi$ and $\tilde{\pi}$ have the same restriction to $K$, so the spaces $\mathcal{H}^{k}$ and the number $s$ are the same for $\pi$ and $\tilde{\pi}$.

Let the moment functional $\mu$, the moments $\mu_{k}$ and the associated bilinear form be defined by (4.1), (4.2) and (4.3), respectively.

PROPOSITION 4.6. The polynomials $p_{k}(k<s)$, defined by (4.8), are the monic orthogonal polynomials with respect to the moment functional $\mu$, i.e. $\mu\left(p_{k} p_{l}\right)=0$ if $k, l<s$ and $k \neq l$.

Proof. If $j<s$ then

$$
\operatorname{span}\left\{e_{0}, \ldots, e_{j}\right\}=\left\{M \text {-invariant vectors in } \mathcal{H}_{j}\right\}=\operatorname{span}\left\{e_{0}, \ldots, \tilde{\pi}\left(\left(i X_{1}\right)^{j}\right) e_{0}\right\} .
$$

If $j<k<s$ then $0=\left\langle e_{k}, e_{j}\right\rangle=\left\langle\pi\left(p_{k}\left(i X_{1}\right)\right) e_{0}, e_{j}\right\rangle$. Hence

$$
0=\left\langle\pi\left(p_{k}\left(i X_{1}\right)\right) e_{0}, \tilde{\pi}\left(\left(i X_{1}\right)^{j}\right) e_{0}\right\rangle=\left\langle\pi\left(\left(i X_{1}\right)^{j} p_{k}\left(i X_{1}\right)\right) e_{0}, e_{0}\right\rangle \text { if } j<k<s .
$$

REMARK 4.7. (a) Note that the moment functional and the polynomials $p_{k}$ are independent of the particular $K$-unitary structure chosen on $\mathcal{H}$. 
(b) For the moment functional $\tilde{\mu}$-and thus for the polynomials $\tilde{p}_{k}$ associated with $\tilde{\pi}$ we have $\tilde{\mu}_{k}=\overline{\mu_{k}}, \tilde{p}_{k}=\overline{p_{k}}$. (Indeed, $\tilde{\mu}_{k}=\overline{\left\langle e_{0}, \tilde{\pi}\left(\left(i X_{1}\right)^{k}\right) e_{0}\right\rangle}=\overline{\left\langle\pi\left(\left(i X_{1}\right)^{k}\right) e_{0}, e_{0}\right\rangle}=\overline{\mu_{k}}$.

(c) The moment functional $\mu$ is symmetric, that is, $\mu_{2 j+1}=0$ for all $j \in \mathbb{Z}_{+}$. (Indeed, take $k \in K$ with $\operatorname{Ad}(k) X_{1}=-X_{1}$ and use $\left.\left\langle\pi\left(\left(i X_{1}\right)^{j}\right) e_{0}, e_{0}\right\rangle=\left\langle\pi\left(\operatorname{Ad}(k)\left(i X_{1}\right)^{j}\right) e_{0}, e_{0}\right\rangle.\right)$

The proof of Theorem 4.1 is completed with

PROPOSITION 4.8. Let $k \in \mathbb{Z}_{+}$. Then the bilinear form $\langle., .\rangle_{\mu}$ is non-degenerate on the space of polynomials of degree $\leq k$ iff $k<s$.

PROOF. If $s<\infty$ then, by Proposition 4.5,

$$
\left\langle p_{s}, q\right\rangle_{\mu}=\left\langle\pi\left(q\left(i X_{1}\right)\right) \pi\left(p_{s}\left(i X_{1}\right)\right) e_{0}, e_{0}\right\rangle=0
$$

for all polynomials $q$. On the other hand, if $k<s \leq \infty$ and $p$ is a polynomial of degree $\leq k$ such that $\langle p, q\rangle_{\mu}=0$ for all polynomials $q$ of degree $\leq k$, then, by Proposition 4.5 and Remark 4.7(b),

$$
\pi\left(p\left(i X_{1}\right)\right) e_{0}=\tilde{\pi}\left(\bar{q}\left(i X_{1}\right)\right) e_{0}
$$

for a certain polynomial $q$ of degree $\leq k$, whence

$$
0=\left\langle\pi\left(q\left(i X_{1}\right) p\left(i X_{1}\right)\right) e_{0}, e_{0}\right\rangle=\left\langle\pi\left(i X_{1}\right) e_{0}, \pi\left(i X_{1}\right) e_{0}\right\rangle .
$$

Hence $\pi\left(p\left(i X_{1}\right)\right) e_{0}=0$ and, again by Proposition $4.5, p=0$.

REMARK 4.9. It follows from Proposition 4.8 that $\left\langle p_{k}, p_{k}\right\rangle_{\mu} \neq 0$ if $k<s$. Thus, if $s=\infty$, then the polynomials $p_{k}$ are orthogonal with respect to the moment functional $\mu$ in the sense of Chihara [3, Chapter I, Definition 2.2].

Proof of Theorem 4.2. Assume $\pi$ unitary. Then for $0 \neq\left(c_{1}, \ldots, c_{k}\right) \in \mathbb{C}^{k}, k<s$, we have by Proposition 4.5 that

$$
\begin{aligned}
0 & <\left\langle\pi\left(\sum_{p \leq k} c_{p}\left(i X_{1}\right)^{p}\right) e_{0}, \pi\left(\sum_{q \leq k} c_{q}\left(i X_{1}\right)^{q}\right) e_{0}\right\rangle \\
& =\sum_{p, q \leq k} c_{p} \overline{c_{q}}\left\langle\pi\left(\left(i X_{1}\right)^{p+q}\right) e_{0}, e_{0}\right\rangle=\sum_{p, q \leq k} c_{p} \overline{c_{q}} \mu_{p+q} .
\end{aligned}
$$

Conversely assume that $\sum_{p, q \leq k} c_{p} \overline{c_{q}} \mu_{p+q}>0$ whenever $k<s$ and $0 \neq\left(c_{1}, \ldots, c_{k}\right) \in \mathbb{C}^{k}$. Use that

$$
\left\langle e_{k}, \tilde{\pi}\left(p_{j}\left(i X_{1}\right)\right) e_{0}\right\rangle=\left\langle\pi\left(p_{k}\left(i X_{1}\right)\right) e_{0}, \tilde{\pi}\left(p_{j}\left(i X_{1}\right)\right) e_{0}\right\rangle=\left\langle\pi\left(\overline{p_{j}}\left(i X_{1}\right) p_{k}\left(i X_{1}\right)\right) e_{0}, e_{0}\right\rangle .
$$

Hence

$$
\left\langle e_{k}, \tilde{\pi}\left(p_{j}\left(i X_{1}\right)\right) e_{0}\right\rangle=\alpha_{k} \delta_{j, k} \text { for all } j, k<s, \text { where } \alpha_{k}>0(0 \leq k<s) .
$$

Also, by Proposition 4.5,

$$
\left\langle e_{k}, \pi\left(p_{j}\left(i X_{1}\right)\right) e_{0}\right\rangle=\beta_{k} \delta_{j, k} \text { for all } j, k<s, \text { where } \beta_{k}>0(0 \leq k<s) .
$$


$:=\alpha_{k} / \beta_{k}>0$. Then

$$
\left\langle e_{k}, \tilde{\pi}\left(p\left(i X_{1}\right)\right) e_{0}\right\rangle=\gamma_{k}\left\langle e_{k}, \pi\left(p\left(i X_{1}\right)\right) e_{0}\right\rangle
$$

$k<s$ and all polynomials $p$ of degree $<s$, in particular for $p(x):=x^{j}, j<s$. :

$$
\left\langle e_{k}, \tilde{\pi}(a) e_{0}\right\rangle=\gamma_{k}\left\langle e_{k}, \pi(a) e_{0}\right\rangle
$$

$\equiv A, k<s$. Since $\tilde{\pi}(a) e_{0}$ and $\pi(a) e_{0}$ are $M$-invariant, it follows that

$$
\left\langle v, \tilde{\pi}(a) e_{0}\right\rangle=\gamma_{k}\left\langle v, \pi(a) e_{0}\right\rangle
$$

$\equiv A, k<s, v \in \mathcal{H}^{k}$. Finally, by Cartan decomposition of $G$,

$$
\left\langle\nu, \tilde{\pi}(g) e_{0}\right\rangle=\gamma_{k}\left\langle\nu, \pi(g) e_{0}\right\rangle
$$

$\in G, k<s, v \in \mathcal{H}^{k}$. Now it follows quickly that the spherical function $g \longmapsto$ $\left.2_{0}, e_{0}\right\rangle$ is positive definite on $G$, so $\pi$ is unitarizable. (See also [16, Theorem 6.5 and rk 6.6].)

Relationship between moment functional and spherical function. We prethe notation of the beginning of $\S 4$. Let $\phi$ be the spherical function associated with presentation $\pi$, i.e.

$$
\phi(x):=\left\langle\pi(x) e_{0}, e_{0}\right\rangle, \quad x \in G
$$

$\phi$ is a real analytic function on $G$. Each spherical function for the Gelfand pair ) can be obtained from a unique irreducible representation $\pi$ of $G$, see [14, TheoV.4.5].

:OPOSITION 5.1. In terms of the spherical function $\phi$ the moments can be expressed

$$
\mu_{k}=\left.(i d / d t)^{k}\right|_{t=0} \phi\left(\exp \left(t X_{1}\right)\right)
$$

:OOF.

$\left.\pi\left(\left(i X_{1}\right)^{k}\right) e_{0}, e_{0}\right\rangle=\left.(i d / d t)^{k}\right|_{t=0}\left\langle\pi\left(\exp \left(t X_{1}\right)\right) e_{0}, e_{0}\right\rangle=\left.(i d / d t)^{k}\right|_{t=0} \phi\left(\exp \left(t X_{1}\right)\right)$.

order to associate a weight function with the moment functional $\mu$ in (5.1) we can r Fourier techniques to this formula. 
PROPOSITION 5.2. Suppose that the function $t \longmapsto \phi\left(\exp \left(t X_{1}\right)\right)$ is of the form

$$
\phi\left(\exp \left(t X_{1}\right)\right)=\sum_{j=1}^{r} \omega_{j} e^{-i \zeta j t}+\int_{-\infty}^{\infty} \omega(\xi) e^{-i \xi t} d \xi
$$

where $\zeta_{1}, \ldots, \zeta_{r} \in \mathbb{C}$ and distinct, $\omega_{1}, \ldots, \omega_{r} \in \mathbb{C}$ and nonzero, and $\xi \mapsto \xi^{k} \omega(\xi)$ is in $L^{1}(\mathbb{R})$ for all $k \in \mathbb{Z}_{+}$. Then

$$
\mu_{k}=\sum_{j=1}^{r} \zeta_{j}^{k} \omega_{j}+\int_{-\infty}^{\infty} \xi^{k} \omega(\xi) d \xi, \quad k \in \mathbb{Z}_{+},
$$

and the polynomials $p_{k}$ are monic orthogonal polynomials with respect to the orthogonality measure

$$
\left(\omega(\xi)+\sum_{j=1}^{r} \omega_{j} \delta\left(\xi-\zeta_{j}\right)\right) d \xi
$$

ProOF. Substitute (5.2) into (5.1).

Later in this section we will see when the conditions of the above proposition are satisfied.

REMARK 5.3. If $r=0$ in (5.2) then the function $t \mapsto \phi\left(\exp \left(t X_{1}\right)\right)$ is the classical Fourier transform of $\omega$. In general, (5.2) writes the function $t \mapsto \phi\left(\exp \left(t X_{1}\right)\right)$, when identified with a distribution in $\mathcal{D}^{\prime}(\mathbb{R})$, as the Fourier transform of $\omega+\sum_{j=1}^{r} \omega_{j} \delta\left(.-\zeta_{j}\right)$ considered as the linear functional

$$
\psi \mapsto \sum_{j=1}^{r} \psi\left(\zeta_{j}\right) \omega_{j}+\int_{-\infty}^{\infty} \psi(\xi) \omega(\xi) d \xi
$$

on the space of Fourier transforms of $\mathcal{D}(\mathbb{R})$ ( $c f$. Gelfand and Shilov [12, Chapter II, Sections 2.1, 2.2]). If $\phi\left(\exp \left(t X_{1}\right)\right)$ can be expressed in the form of (5.2) then the function $\omega$ and the pairs $\left(\omega_{k}, \zeta_{k}\right)$ are uniquely determined by $\phi$.

PROPOSITION 5.4. Suppose that $\phi$ can be written in the form (5.2). Then $\omega \neq 0$ iff $s=\infty$. If $\omega=0$ then $s=r$.

Proof. If $s<\infty$ then, by Proposition 4.5,

$$
0=\pi\left(p_{s}\left(i X_{1}\right)\right) \phi\left(\exp \left(t X_{1}\right)\right)=p_{s}(i d / d t) \phi\left(\exp \left(t X_{1}\right)\right) \text { for all } t \in \mathbb{R} .
$$

It follows from (5.2) that then $\omega=0$ and $r \leq s$.

On the other hand, if $\phi$ can be written in the form (5.2) with $\omega=0$ then

$$
p(i d / d t) \phi\left(\exp \left(t X_{1}\right)\right)=0 \text { with } p(x)=\prod_{j=1}^{r}\left(x-\zeta_{j}\right) .
$$

Hence $\langle p, q\rangle_{\mu}=0$ for all polynomials $q$ of degree $\leq r$, so $r \geq s$ by Proposition 4.8. 
Now we will determine explicitly the polynomials $p_{k}$ in the various cases. Put

$$
\alpha:=d / 2-1 \text {. }
$$

5.1 The Euclidean case $G=I\left(\mathbb{R}^{d}\right)$. The spherical functions are given by

$$
\phi\left(\exp \left(t X_{1}\right)\right)=j_{\alpha}(\lambda t)
$$

essentially a Bessel function ( $c f$. (2.10)), where $\lambda$ runs over $C$ with $\lambda$ and $-\lambda$ identified, see Faraut [9, Section II.II]. The corresponding representations are unitarizable iff $\lambda \in \mathbb{R}$. If $\lambda=0$ then $\pi$ is the identity representation and $s=1$. If $\lambda \in \mathbb{C} \backslash\{0\}$ then $\phi\left(\exp \left(t X_{1}\right)\right)$ has the form (5.2) with $r=0$, explicitly given by (2.11). It follows from (2.11) that the corresponding monic polynomials $p_{k}$ are given in terms of Gegenbauer polynomials as

$$
p_{k}(x)=\frac{\lambda^{k}(2 \alpha)_{k}}{2^{k}(\alpha)_{k}} R_{k}^{\left(\alpha-\frac{1}{2}, \alpha-\frac{1}{2}\right)}(x / \lambda) .
$$

5.2 The noncompact case $G=O_{0}(1, d)$. We assume sectional curvature -1 in $O_{0}(1, d) /$ $O(d)$. Then the spherical functions are given by

$$
\phi\left(\exp \left(t X_{1}\right)\right)=\phi_{\lambda}^{\left(\alpha,-\frac{1}{2}\right)}(t),
$$

a Jacobi function ( $c f .(2.1)$ ), where $\lambda$ runs over $\mathbb{C}$ with $\lambda$ and $-\lambda$ identified, see for instance Faraut [9, Sections II.IV and IV.I]. The corresponding representations are unitarizable iff $\lambda \in \mathbb{R}$ or $-\alpha \leq i \lambda \leq \alpha$. Because of (2.3), the function $t \mapsto \phi\left(\exp \left(t X_{1}\right)\right)$ belongs to the class $S$ if $|\operatorname{Im} \lambda|<\alpha+\frac{1}{2}$. Then (5.2) holds with $r=0$ and we get (5.2) explicitly from (2.6). In combination with Proposition 5.2 the corresponding polynomials $p_{k}$ are seen to be expressible in terms of continuous symmetric Hahn polynomials by (2.13):

$$
p_{k}(x):=\frac{(-1)^{k}(2 \alpha)_{k}\left(\frac{1}{2}+\alpha+i \lambda\right)_{k}}{2^{k}(\alpha)_{k}} p_{k}\left(\frac{x}{2} ; \frac{1}{4}+\frac{\alpha}{2}+\frac{i \lambda}{2}, \frac{1}{4}+\frac{\alpha}{2}-\frac{i \lambda}{2}\right) .
$$

By analytic continuation with respect to $\lambda$ this formula remains correct for all $\lambda \in \mathbb{C}$. For $\lambda \notin i \mathbb{Z}, t \neq 0$, we have an expansion of the form

$$
\phi_{\lambda}^{\left(\alpha,-\frac{1}{2}\right)}(t)=\sum_{j=0}^{\infty}\left(c_{j}(-\lambda) e^{\left(-i \lambda-\left(\alpha+\frac{1}{2}+2 j\right)\right)|t|}+c_{j}(\lambda) e^{\left(i \lambda-\left(\alpha+\frac{1}{2}+2 j\right)\right)|t|}\right)
$$

(see [17, (2.17), (2.15)]). Hence, if $\operatorname{Im} \lambda \geq 0, \operatorname{Im} \lambda-\alpha-\frac{1}{2} \notin 2 \mathbb{Z}_{+}$and $-i \lambda \notin \mathbb{Z}_{+}$then

$$
\phi_{\lambda}^{\left(\alpha,-\frac{1}{2}\right)}(t)=\sum_{0 \leq 2 j<\operatorname{Im} \lambda-\alpha-\frac{1}{2}} 2 c_{j}(-\lambda) \cosh \left(\left(i \lambda+\alpha+\frac{1}{2}+2 j\right) t\right)+\psi_{\lambda}(t),
$$

where $\psi_{\lambda} \in S$. Thus, for these $\lambda$, the function $\phi$ has the form (5.2).

If $-i \lambda-\alpha-\frac{1}{2}=n, n \in \mathbb{Z}_{+}$then, by the quadratic transformation [17, (5.32)], by (2.1) and $(2.7)$ we have that

$$
\phi_{\lambda}^{\left(\alpha,-\frac{1}{2}\right)}(t)=R_{n}^{(\alpha, \alpha)}(\cosh t)
$$


a Jacobi polynomial of argument $\cosh t, c f$. (2.7). Then $\phi$ is of the form (5.2) with $\omega=0$, so the corresponding representation of $G$ extends by analytic continuation to a representation of the complexification $O_{0}(d+1, \mathbb{C}$ ) and can next be restricted to a (still spherical and irreducible) representation of the compact form $O(d+1)$. The spherical function for $(O(d+1), O(d))$ keeps the same expression as above, except that $\cosh t$ is replaced by $\cos t$.

5.3 The compact case $G=O(d+1)$. We assume curvature +1 on $O(d+1) / O(d)$. Then the spherical functions are given by

$$
\phi\left(\exp \left(t X_{1}\right)\right)=R_{n}^{(\alpha, \alpha)}(\cos t), \quad n \in \mathbb{Z}_{+},
$$

up to a constant factor a Jacobi polynomial (cf. (2.7)). See Vilenkin [23, Chapter 9]. Since $G$ is compact, the corresponding representations are all unitarizable. From (2.8) we see that $\phi\left(\exp \left(t X_{1}\right)\right)$ has the form (5.2) with $\omega=0$ and $\left\{\zeta_{1}, \ldots, \zeta_{r}\right\}=\{-n,-n+2, \ldots, n-$ $2, n\}$. Hence $s=n+1$ by Proposition 5.4. By Proposition 5.2 and (2.15) we get that the corresponding polynomials $p_{k}$ are given in terms of Hahn polynomials by (2.15):

$$
p_{k}(x)=\frac{(-1)^{k}(2 \alpha)_{k}(-n)_{k}}{2^{k}(\alpha)_{k}} Q_{k}\left(\frac{1}{2}(n-x) ; \alpha-\frac{1}{2}, \alpha-\frac{1}{2}, n\right) .
$$

6. Associated spherical functions. We still preserve the notation of Section 4. For the given spherical representation $\pi$ on $\mathcal{H}$ and $k \in \mathbb{Z}_{+}$define the vectors $\tilde{e}_{k}$ corresponding to $\tilde{\pi}$ similarly to (4.7), (4.8):

$$
\tilde{e}_{k}:=\tilde{\pi}\left(\sigma\left(P_{k}\left(i X_{1}, \ldots, i X_{d}\right)\right)\right) e_{0}=\tilde{\pi}\left(\overline{p_{k}}\left(i X_{1}\right)\right) e_{0}
$$

(cf. Remark 4.7). Then $\tilde{e}_{0}=e_{0}, \tilde{e}_{k}=0$ iff $k \geq s$ and $\tilde{e}_{k}$ is proportional to $e_{k}$ for all $k\left(\tilde{e}_{k}\right.$ is equal to $e_{k}$ for all $k$ iff $\pi$ is unitary, $c f$. the end of the proof of Theorem 4.2). Note that we have normalized the vectors $\tilde{e}_{k}$ such that the constants

(6.2) $\gamma_{k}:=\left\langle e_{k}, \tilde{e}_{k}\right\rangle=\left\langle\pi\left(p_{k}\left(i X_{1}\right)\right) e_{0}, \tilde{\pi}\left(\overline{p_{k}}\left(i X_{1}\right)\right) e_{0}\right\rangle=\left\langle\pi\left(p_{k}^{2}\left(i X_{1}\right)\right) e_{0}, e_{0}\right\rangle=\left\langle p_{k}, p_{k}\right\rangle_{\mu}$

depend only on the representation $\pi$, but not on the particular $K$-unitary structure chosen on $\mathcal{H}$ ( $c f$. Remark 4.7(a)). For instance, it follows from Theorem 4.2 that $\pi$ is unitarizable if and only if the $\gamma_{k}$ are positive real for all $k<s$.

Define for $k \in \mathbb{Z}_{+}$the associated spherical function $\phi^{k}$ on $G$ by

$$
\phi^{k}(g):=\left\langle\pi(g) e_{0}, \tilde{e}_{k}\right\rangle
$$

(See [17, Section 3.3] for generalities on associated spherical functions.) Each function $\phi^{k}$ is real analytic on $G$, left $M$ - and right $K$-invariant. 
PROPOSITION 6.1. The associated spherical function $\phi^{k}$ is obtained from the spherical function $\phi$ by

$$
\phi^{k}(g)=\left(p_{k}\left(i X_{1}\right) \phi\right)(g) .
$$

Here $X_{1}$ is the right invariant differential operator associated with the Lie algebra element $X_{1}$, i.e. $\left(X_{1} f\right)(g):=\left.(d / d t)\right|_{t=0} f\left(\exp \left(t X_{1}\right) g\right)$. In particular, for $g=\exp t X_{1}$,

$$
\phi^{k}\left(\exp t X_{1}\right)=p_{k}(i d / d t) \phi\left(\exp t X_{1}\right) .
$$

The function $t \mapsto \phi^{k}\left(\exp t X_{1}\right)$ has a zero of order $k$ at 0 and for $k<s$ it is specified by this property among the complex linear combinations

$$
(i d / d t)^{k} \phi\left(\exp t X_{1}\right)+c_{k-1}(i d / d t)^{k-1} \phi\left(\exp t X_{1}\right)+\cdots+c_{0} \phi\left(\exp t X_{1}\right) .
$$
$\left.e_{0}\right\rangle$.

ProOF. (i) $\phi^{k}(g)=\left\langle\pi(g) e_{0}, \tilde{e}_{k}\right\rangle=\left\langle\pi(g) e_{0}, \tilde{\pi}\left(\overline{p_{k}}\left(i X_{1}\right)\right) e_{0}\right\rangle=\left\langle\pi\left(p_{k}\left(i X_{1}\right)\right) \pi(g) e_{0}\right.$,

(ii) $\left.(d / d t)^{j}\right|_{t=0} \phi^{k}\left(\exp t X_{1}\right)=\left\langle\pi\left(\left(X_{1}\right)^{j}\right) e_{0}, \tilde{e}_{k}\right\rangle=0$ for all $j<k$ by Proposition 4.5. The converse statement follows analogously from Proposition 4.6.

From Proposition 6.1 it follows that the associated spherical functions $\phi^{k}$ do not depend on the particular $K$-unitary structure chosen on $\mathcal{H}$. Furthermore, $\phi^{k}$ is determined by its restriction to the one-parameter subgroup $A$ of $G$. To see this, write $g=k_{1} a k_{2}$ $\left(k_{1}, k_{2} \in K, a \in A\right)$ by the Cartan decomposition. Then

$$
\phi^{k}\left(k_{1} a k_{2}\right)=\left\langle\pi(a) e_{0}, \tilde{\pi}\left(k_{1}^{-1}\right) \tilde{e}_{k}\right\rangle=\left\langle\pi(a) e_{0}, \tilde{e}_{k}\right\rangle \frac{\left\langle\pi\left(k_{1}\right) \tilde{e}_{k}, \tilde{e}_{k}\right\rangle}{\left\langle\tilde{e}_{k}, \tilde{e}_{k}\right\rangle}=\phi^{k}(a) \psi^{k}\left(k_{1}\right)
$$

since $\pi(a) e_{0}$ is $M$-invariant, $\tilde{\pi}\left(k_{1}^{-1}\right) \tilde{e}_{k}$ is in $\mathcal{H}^{k}$ and, up to a constant, $\tilde{e}_{k}$ is the unique $M$-invariant vector in $\mathcal{H}^{k}$. The function $\psi^{k}$ is the spherical function for the pair $(K, M)$ associated to the representation $\left.\pi\right|_{\mathcal{H}^{k}}$ of $K$. It does not depend on the representation $\pi$, neither on the type of the group $G$ (only on the dimension $d$ ). Recall the definition of $\operatorname{Ad}(K)$-invariant inner product on ${ }^{\ddagger}$ given in the beginning of Section 4 . It induces an $\operatorname{Ad}(M)$-invariant inner product on the orthoplement in ${ }^{f}$ to the Lie algebra of $M$ such that the corresponding sphere $K / M$ has sectional curvature 1. Let $Y_{1}$ be a unit vector in this orthoplement. Put $k_{\theta}=\exp \theta Y_{1}$. By formula (5.5) we have

$$
\psi^{k}\left(k_{\theta}\right)=R_{k}^{\left(\alpha-\frac{1}{2}, \alpha-\frac{1}{2}\right)}(\cos \theta) .
$$

Up to a constant factor, the associated spherical functions $t \mapsto \phi^{k}\left(\exp t X_{1}\right)$ are known as special functions on $\mathbb{R}$ in all cases (for instance they can be found as solutions of singular second order differential equations on $\mathbb{R}$ ). We shall determine the proper normalization in all cases. From the function $\phi^{k}$ the constant $\gamma_{k}$ can be calculated as

$$
\gamma_{k}=\left.p_{k}(i d / d t)\right|_{t=0} \phi^{k}\left(\exp t X_{1}\right)=\left.(i d / d t)^{k}\right|_{t=0} \phi^{k}\left(\exp t X_{1}\right) .
$$


6.1 The Euclidean case $G=I\left(\mathbb{R}^{d}\right)$. The functions $\phi^{k}$ associated to the spherical function $\phi\left(\exp t X_{1}\right)=j_{\alpha}(\lambda t)$ are of the form

$$
\phi^{k}\left(\exp t X_{1}\right)=\text { const. } t^{k} j_{\alpha+k}(\lambda t)
$$

(see [23, Section XI.3.3]). Hence, for $\alpha=-\frac{1}{2}, 0, \frac{1}{2}, \ldots$, formula (6.4) provides us with a group theoretic interpretation of formula (3.6). Setting $g=k_{\theta} \exp t X_{1}$ in formula (6.3) leads, in view of (6.5), to a group theoretic interpretation of formula (3.5).

The correct normalization of the associated spherical function $\phi^{k}$ can be read off from formula (3.6):

$$
\phi^{k}\left(\exp t X_{1}\right)=p_{k}(i d / d t) j_{\alpha}(\lambda t)=\frac{(-i)^{k}(2 \alpha)_{k} \lambda^{2 k}}{4^{k}(\alpha)_{k}(\alpha+1)_{k}} t^{k} j_{\alpha+k}(\lambda t)
$$

with $p_{k}$ given by (5.3). Hence, by formula (6.6),

$$
\gamma_{k}=\frac{(2 \alpha)_{k} k !}{4^{k}(\alpha)_{k}(\alpha+1)_{k}} \lambda^{2 k} .
$$

6.2 The noncompact case $G=O_{0}(1, d)$. The functions $\phi^{k}$ associated to the spherical function $\phi\left(\exp t X_{1}\right)=\phi_{\lambda}^{\left(\alpha,-\frac{1}{2}\right)}(t)$ are of the form

$$
\phi^{k}\left(\exp t X_{1}\right)=\text { const. }(\sinh t)^{k} \phi_{\lambda}^{\left(\alpha+k,-\frac{1}{2}\right)}(t)
$$

(see $[23, \S X .3 .4]$ or $[17, \S 4.2]$ ). First we evaluate the constant. For $\operatorname{Im} \lambda>0, t \rightarrow \infty$ we have

$$
\phi_{\lambda}^{\left(\alpha,-\frac{1}{2}\right)}(t) \sim c_{\alpha}(-\lambda) e^{-\left(i \lambda+\alpha+\frac{1}{2}\right) t} \text { with } c_{\alpha}(\lambda)=\frac{2^{2 \alpha} \Gamma(\alpha+1) \Gamma(i \lambda)}{\sqrt{\pi} \Gamma\left(i \lambda+\alpha+\frac{1}{2}\right)}
$$

(see $[17,(2.18)])$. Thus on the one hand we obtain

$$
\lim _{t \rightarrow \infty} \frac{(\sinh t)^{k} \phi_{\lambda}^{\left(\alpha+k,-\frac{1}{2}\right)}(t)}{\phi_{\lambda}^{\left(\alpha,-\frac{1}{2}\right)}(t)}=\frac{c_{\alpha+k}(-\lambda)}{2^{k} c_{\alpha}(-\lambda)}=\frac{2^{k}(\alpha+1)_{k}}{\left(\alpha+\frac{1}{2}-i \lambda\right)_{k}},
$$

and on the other hand we have

(6.8) $\lim _{t \rightarrow \infty} \frac{\phi^{k}\left(\exp t X_{1}\right)}{\phi\left(\exp t X_{1}\right)}=\lim _{t \rightarrow \infty} \frac{p_{k}(i d / d t) \phi\left(\exp t X_{1}\right)}{\phi\left(\exp t X_{1}\right)}=p_{k}\left(\lambda-i\left(\alpha+\frac{1}{2}\right)\right)$.

By use of formulas (5.4) and (2.12) we can calculate the right hand side of this last formula explicitly:

$$
p_{k}\left(\lambda-i\left(\alpha+\frac{1}{2}\right)\right)=\frac{(-i)^{k}(2 \alpha)_{k}}{2^{k}(\alpha)_{k}}\left(\alpha+\frac{1}{2}+i \lambda\right)_{k} .
$$

Altogether, we obtain explicitly for $\phi^{k}$

(6.10) $\phi^{k}\left(\exp t X_{1}\right)=\frac{(-i)^{k}(2 \alpha)_{k}}{4^{k}(\alpha)_{k}(\alpha+1)_{k}}\left(\alpha+\frac{1}{2}+i \lambda\right)_{k}\left(\alpha+\frac{1}{2}-i \lambda\right)_{k}(\sinh t)^{k} \phi_{\lambda}^{\left(\alpha+k,-\frac{1}{2}\right)}(t)$

and then for $\gamma_{k}$

$$
\gamma_{k}=\frac{(2 \alpha)_{k} k !}{4^{k}(\alpha)_{k}(\alpha+1)_{k}}\left(\alpha+\frac{1}{2}+i \lambda\right)_{k}\left(\alpha+\frac{1}{2}-i \lambda\right)_{k}
$$

By analytic continuation, formulas (6.9)-(6.11) hold for all $\lambda \in \mathbb{C}$. 
IOREM 6.2. For all $\lambda \in \mathbb{C}, k \in \mathbb{Z}_{+}$and $\alpha=0, \frac{1}{2}, 1, \ldots$ we have

$$
\begin{gathered}
p_{k}\left(\frac{i}{2}\left(\cos \theta \frac{\partial}{\partial t}-\operatorname{coth} t \sin \theta \frac{\partial}{\partial \theta}\right) ; \frac{\alpha}{2}+\frac{1}{4}+\frac{i \lambda}{2}, \frac{\alpha}{2}+\frac{1}{4}-\frac{i \lambda}{2}\right) \phi_{\lambda}^{\left(\alpha,-\frac{1}{2}\right)}(t) \\
\quad=\frac{i^{k}}{2^{k}(\alpha+1)_{k}}\left(\alpha+\frac{1}{2}-i \lambda\right)_{k}(\sinh t)^{k} \phi_{\lambda}^{\left(\alpha+k,-\frac{1}{2}\right)}(t) R_{k}^{\left(\alpha-\frac{1}{2}, \alpha-\frac{1}{2}\right)}(\cos \theta)
\end{gathered}
$$

$\downarrow$ in particular, for $\theta=0$,

$$
\begin{aligned}
p_{k}\left(\frac{i}{2} \frac{d}{d t} ; \frac{\alpha}{2}+\frac{1}{4}+\frac{i \lambda}{2}, \frac{\alpha}{2}+\frac{1}{4}-\frac{i \lambda}{2}\right) & \phi_{\lambda}^{\left(\alpha,-\frac{1}{2}\right)}(t) \\
= & \frac{i^{k}}{2^{k}(\alpha+1)_{k}}\left(\alpha+\frac{1}{2}-i \lambda\right)_{k}(\sinh t)^{k} \phi_{\lambda}^{\left(\alpha+k,-\frac{1}{2}\right)}(t) .
\end{aligned}
$$

JoF. We use formulas (5.4) and (6.10) to write down formula (6.4) explicitly; this to formula (6.13) when $\left(\alpha+\frac{1}{2}+i \lambda\right)_{k} \neq 0$; by continuity, formula (6.13) remains 'hen $\left(\alpha+\frac{1}{2}+i \lambda\right)_{k}=0$. To obtain formula (6.12) analogously from formula (6.3), zrely have to show that for any right $K$-invariant function $f \in C^{\infty}(G)$ we have

$$
\left(X_{1} f\right)\left(k_{\theta} \exp t X_{1}\right)=(\cos \theta \partial / \partial t-\operatorname{coth} t \sin \theta \partial / \partial \theta) f\left(k_{\theta} \exp t X_{1}\right) .
$$

that $X_{1}$ and $Y_{1}$ span a Lie algebra isomorphic to the Lie algebra $\operatorname{sl}(2, \mathbb{R})$ and that mages under this isomorphism can be taken, for instance, as

$$
Y_{1}=\left(\begin{array}{cc}
0 & -\frac{1}{2} \\
\frac{1}{2} & 0
\end{array}\right), \quad X_{1}=\left(\begin{array}{cc}
\frac{1}{2} & 0 \\
0 & -\frac{1}{2}
\end{array}\right) .
$$

2

$$
k_{\theta}=\left(\begin{array}{cc}
\cos \frac{1}{2} \theta & -\sin \frac{1}{2} \theta \\
\sin \frac{1}{2} \theta & \cos \frac{1}{2} \theta
\end{array}\right), \quad \exp t X_{1}=\left(\begin{array}{cc}
e^{\frac{1}{2} t} & 0 \\
0 & e^{-\frac{1}{2} t}
\end{array}\right) .
$$

define the functions $r, \psi: \mathbb{R} \rightarrow \mathbb{R}$ by $\exp \left(s X_{1}\right) k_{\theta} \exp \left(t X_{1}\right) \in k_{\psi(s)} \exp \left(r(s) X_{1}\right) K$.

$$
\left(X_{1} f\right)\left(k_{\theta} \exp t X_{1}\right)=(\dot{r}(0) \partial / \partial t+\dot{\psi}(0) \partial / \partial \theta) f\left(k_{\theta} \exp t X_{1}\right)
$$

1 easy calculation we find

$$
\begin{gathered}
\cosh r=\cosh (s+t) \cos ^{2} \frac{1}{2} \theta+\cosh (s-t) \sin ^{2} \frac{1}{2} \theta \\
\sinh r \sin \psi=\sinh t \sin \theta .
\end{gathered}
$$

ifferentiate these two formulas with respect to $s$ at $s=0$ and obtain $\dot{r}(0)=\cos \theta$ $',(0)=-\operatorname{coth} t \sin \theta$.

:e $[18, \S 2]$ for a purely analytic proof of $(6.13)$.

he compact case $G=O(d+1)$. The functions $\phi^{k}$ associated to the spherical function ? $\left.t X_{1}\right)=R_{n}^{(\alpha, \alpha)}(\cos t)$ are of the form

$$
\phi^{k}\left(\exp t X_{1}\right)=\text { const. }(\sin t)^{k} R_{n-k}^{(\alpha+k, \alpha+k)}(\cos t)
$$

[23, §IX.4.1]). The appropriate normalizations as well as the explicit versions of ulas (6.3) and (6.4) can be obtained by analytic continuation from the noncompact Put $\alpha+\frac{1}{2}+i \lambda=-n, n \in \mathbb{Z}_{+}$in formulas (6.12), (6.13), replace $t$ by $i t$ and use ${ }^{\left.\frac{1}{2}\right)}(t)=\phi_{2 \lambda}^{(\alpha, \alpha)}\left(\frac{1}{2} t\right)$ in order to obtain the following corollary. 
COROLLARY 6.3. For all $k, n \in \mathbb{Z}_{+}, k \leq n$ and $\alpha \neq-\frac{1}{2},-1,-\frac{3}{2}, \ldots$ we have

$$
Q_{k}\left(\frac{1}{2} n-\frac{1}{2} i(\cos \theta \partial / \partial t-\cot t \sin \theta \partial / \partial \theta) ; \alpha-\frac{1}{2}, \alpha-\frac{1}{2}, n\right) R_{n}^{(\alpha, \alpha)}(\cos t)
$$

and in particular, for $\theta=0$,

$$
=\frac{i^{k}}{2^{k}(\alpha+1)_{k}}(n+2 \alpha+1)_{k}(\sin t)^{k} R_{n-k}^{(\alpha+k, \alpha+k)}(\cos t) R_{k}^{\left(\alpha-\frac{1}{2}, \alpha-\frac{1}{2}\right)}(\cos \theta)
$$

$$
\begin{aligned}
Q_{k}\left(\frac{1}{2} n-\frac{1}{2} i d / d t ; \alpha-\frac{1}{2}, \alpha\right. & \left.-\frac{1}{2}, n\right) R_{n}^{(\alpha, \alpha)}(\cos t) \\
= & \frac{i^{k}}{2^{k}(\alpha+1)_{k}}(n+2 \alpha+1)_{k}(\sin t)^{k} R_{n-k}^{(\alpha+k, \alpha+k)}(\cos t) .
\end{aligned}
$$

PROOF. For $\alpha=0, \frac{1}{2}, 1, \ldots$ equation (6.14) is obtained by analytic continuation from formula (6.12). Now fix $n, k$ and $t$. Then both sides of (6.14) are rational functions of $\alpha$ (see formulas (2.14) and (2.7)). Hence the two sides must agree for all $\alpha$.

7. Associated intertwining functions. Again we assume the notation of Section 4. Let $f$ be a generalized left $K$-invariant matrix element of the spherical representation $\pi$ of $G$, i.e.

$$
f(g)=\left\langle\pi(g) e, e_{0}\right\rangle=\left\langle e, \tilde{\pi}\left(g^{-1}\right) e_{0}\right\rangle,
$$

where $e \in \mathcal{H}^{-\infty}(\pi)$, the anti-dual of the Fréchet space $\mathcal{H}^{\infty}(\tilde{\pi})$ of $C^{\infty}$-vectors for $\tilde{\pi}$. Denote the corresponding $M$-invariant matrix element of higher $K$-type by $f^{k}$,

$$
f^{k}=\left\langle\pi(g) e, \tilde{e}_{k}\right\rangle
$$

Then all the functions $f^{k}$ are real analytic on $G$, and formula (6.3) and its proof generalize to the family of functions $f^{k}$ :

$$
f^{k}(g)=p_{k}\left(i X_{1}\right) f(g)
$$

In the case $e$ is invariant with respect to the action of some closed subgroup $G^{\prime}$ of $G$, the function $f=f^{0}$ is called an intertwining function on the homogeous space $G / G^{\prime}$ and the functions $f^{k}$ are called associated intertwining functions on $G / G^{\prime}$. We shall derive in this section the explicit versions of formula (7.2) for two particular homogenous spaces of the group $G=O_{0}(1, d)$.

Let $G=K A N$ be the Iwaswawa decomposition of $G=O_{0}(1, d)$ associated to the positive Weyl chamber $\mathbb{R}_{+} X_{1}$. Write $H(g)=t$ if $g=k \exp \left(t X_{1}\right) n$ in this decomposition. Let $\pi_{\lambda}$ be the spherical principal series representation corresponding to $\lambda$, i. e., $\pi_{\lambda}$ is left action of $G$ on the space

$$
\mathcal{H}_{\lambda}=\left\{f: G \rightarrow \mathbb{C}\left|f\left(g m \exp \left(t X_{1}\right) n\right)=e^{-\left(i \lambda+\alpha+\frac{1}{2}\right) t} f(g), f\right|_{K} \in L^{2}(K)\right\} .
$$

The $K$-unitary structure on $\mathcal{H}_{\lambda}$ is given by the scalar product $\langle f, h\rangle=\int_{K} f(k) \bar{h}(k) d k$. It follows that $\tilde{\pi}_{\lambda}=\pi_{\bar{\lambda}}$ (use [14, Lemma I.5.19]). If $\lambda$ is not degenerate, i.e. if $\lambda \notin$ 
$\pm i\left(\alpha+\frac{1}{2}+\mathbb{Z}_{+}\right)$, then $\pi_{\lambda}$ is spherical. If $\lambda=i\left(\alpha+\frac{1}{2}+n\right)$ with $n \in \mathbb{Z}_{+}$, then $\pi_{\lambda}$ contains a finite-dimensional spherical subrepresentation with $s=n+1$. The $K$-fixed vector $e_{0} \in \mathcal{H}_{\lambda}$ is the function

$$
e_{0}(g)=e^{-\left(i \lambda+\alpha+\frac{1}{2}\right)(H(g))}
$$

and the corresponding spherical function is $\phi_{\lambda}\left(\exp t X_{1}\right)=\phi_{\lambda}^{\left(\alpha,-\frac{1}{2}\right)}(t)$. In this realization of $\pi_{\lambda}$ on $\mathcal{H}_{\lambda}$ we can identify $\mathcal{H}^{\infty}\left(\pi_{\lambda}\right)$ with the subspace $\mathcal{H}_{\lambda}^{\infty}:=\left\{f \in \mathcal{H}_{\lambda}|f|_{K} \in C^{\infty}(K)\right\}$ of $\mathcal{H}_{\lambda}, c f$. Wallach [24, Theorem 1.8.4] and Dixmier and Malliavin [4].

Fix $\lambda \in \mathbb{C}$. Let $\mathcal{E}_{\lambda}$ be the eigenspace of the Laplace-Beltrami operator $\Delta$ on $G / K$ that contains the spherical function $\phi_{\lambda}$ and let $\mathcal{E}_{\lambda}^{*}$ be the subspace of functions of at most exponential growth (see Flensted-Jensen [11, Section IV.2]). Suppose $\lambda \notin i\left(\alpha+\frac{1}{2}+\mathbb{Z}_{+}\right)$; then each function $f \in \mathcal{E}_{\lambda}^{*}$ can be represented as Poisson transform of a distribution $\bar{T}$ on $G$ satisfying $\bar{T}\left(g m \exp t X_{1} n\right)=e^{(i \lambda-\alpha-1 / 2) t} \bar{T}(g)$ (see [11, Corollary IV.6]):

$$
f(g)=P_{\lambda} \bar{T}(g)=\int_{K} e_{0}\left(g^{-1} k\right) \bar{T}(k) d k=\left\langle\pi_{\lambda}(g) e_{0}, e\right\rangle,
$$

where $e \in \mathcal{H}^{-\infty}\left(\pi_{\bar{\lambda}}\right)$ is defined by

$$
\langle e, v\rangle=\int_{K} \overline{v(k)} T(k) d k, \quad v \in \mathcal{H}_{\lambda}^{\infty}=\mathcal{H}^{\infty}\left(\pi_{\lambda}\right)
$$

Thus we find:

LEMMA 7.1. Let $\lambda \notin i\left(\alpha+\frac{1}{2}+\mathbb{Z}_{+}\right)$. Then each $f \in \mathcal{E}_{\lambda}^{*}$ can be written as

$$
f(g)=\left\langle\pi_{\lambda}(g) e_{0}, e\right\rangle=\overline{\left\langle\pi_{\bar{\lambda}}\left(g^{-1}\right) e, e_{0}\right\rangle} .
$$

for a certain e $\in \mathcal{H}^{-\infty}\left(\pi_{\bar{\lambda}}\right)$.

In the degenerate case, a vector distribution of the finite dimensional spherical representation $\pi$ is just an ordinary element of the representation space.

7.1 Associated intertwining functions on the space $G / M N$. The subspace of the $M N$ invariant functions in $\mathcal{E}_{\bar{\lambda}}$ is spanned by the two functions $f_{ \pm}$given explicitly by

$$
f_{ \pm}(g)=e^{-( \pm i \bar{\lambda}+\alpha+1 / 2) H\left(g^{-1}\right)},
$$

see e.g. [14, Chapter 2$],[17, \S 4.1]$. If $\lambda$ is nondegenerate, by formula (7.3) the functions $f_{ \pm}$can be represented as generalized matrix elements of $\pi_{\lambda}$ with two $M N$-invariant elements $e_{ \pm}$of $\mathcal{H}^{-\infty}\left(\pi_{\lambda}\right)$,

$$
f_{ \pm}(g)=\overline{\left\langle\pi_{\lambda}\left(g^{-1}\right) e_{ \pm}, e_{0}\right\rangle} .
$$

Now define the functions $\phi_{ \pm}(g)=\overline{f_{ \pm}\left(g^{-1}\right)}$. Then $\phi_{ \pm}$are explicitly given by

$$
\phi_{ \pm}\left(k \exp \left(t X_{1}\right) n\right)=e^{( \pm i \lambda-\alpha-1 / 2) t} \quad(k \in K, t \in \mathbb{R}, n \in N),
$$

and are intertwining functions on $G / M N$,

$$
\phi_{ \pm}(g)=\left\langle\pi_{\lambda}(g) e_{ \pm}, e_{0}\right\rangle .
$$


(See also Strasburger [21].) From formulas (7.2) and (7.4) we can calculate also the associated intertwining functions $\phi_{ \pm}^{k}$ explicitly:

$$
\phi_{ \pm}^{k}\left(\exp t X_{1}\right)=p_{k}\left(\mp \lambda-i\left(\alpha+\frac{1}{2}\right)\right) e^{( \pm i \lambda-\alpha-1 / 2) t} .
$$

Next, similarly to (6.5), we derive

$$
\phi_{ \pm}^{k}\left(k_{\theta} \exp t X_{1} n\right)=\phi_{ \pm}^{k}\left(\exp \left(t X_{1}\right)\right) \psi^{k}\left(k_{\theta}\right)=p_{k}\left(\mp \lambda-i\left(\alpha+\frac{1}{2}\right)\right) e^{( \pm i \lambda-\alpha-1 / 2) t} \psi^{k}\left(k_{\theta}\right) .
$$

Incidentally, we see from formulas (7.5) and (6.9) that in the case of degenerate $\pi$, where $\lambda=i\left(\alpha+\frac{1}{2}+n\right), s=n+1\left(n \in \mathbb{Z}_{+}\right), e_{+}$is not an element of $\mathcal{H}^{-\infty}(\pi)$, since then $p_{s}\left(-\lambda-i\left(\alpha+\frac{1}{2}\right)\right) \neq 0$. But $p_{s}\left(\lambda-i\left(\alpha+\frac{1}{2}\right)\right)=0$, so we don't have this obstruction for $e_{-}$, and indeed $e_{-} \in \mathcal{H}(\pi)$ is then just the highest weight vector of the finite dimensional representation $\pi$.

PROPOSITION 7.2. For all $\lambda \in \mathbb{C}, k \in \mathbb{Z}_{+}$and $\alpha \neq-\frac{1}{2},-1,-\frac{3}{2}, \ldots$ we have

$$
\begin{gathered}
p_{k}\left(\frac{i}{2}\left(\cos \theta \frac{\partial}{\partial t}-\sin \theta \frac{\partial}{\partial \theta}\right) ; \frac{\alpha}{2}+\frac{1}{4}+\frac{i \lambda}{2}, \frac{\alpha}{2}+\frac{1}{4}-\frac{i \lambda}{2}\right) e^{(i \lambda-\alpha-1 / 2) t} \\
=i^{k} \frac{\left(\alpha+\frac{1}{2}-i \lambda\right)_{k}}{\left(\alpha+\frac{1}{2}+i \lambda\right)_{k}} e^{(i \lambda-\alpha-1 / 2) t} R_{k}^{(\alpha-1 / 2, \alpha-1 / 2)}(\cos \theta) .
\end{gathered}
$$

PROOF. The proof is analogous to the proof of Theorem 6.2. First we show the formula for $\theta=0$, by using (2.13) and (6.9). Next we find for right $M N$-invariant functions $f$ on $G$ that

$$
\left(X_{1} f\right)\left(k_{\theta} \exp t X_{1}\right)=\left(\cos \theta \frac{\partial}{\partial t}-\sin \theta \frac{\partial}{\partial \theta}\right) f\left(k_{\theta} \exp t X_{1}\right) .
$$

This proves the proposition for $\alpha=0, \frac{1}{2}, 1, \ldots$ Now multiply both sides of (7.6) by $e^{-\left(i \lambda-\alpha-\frac{1}{2}\right) t}$ and fix $\lambda, k$ and $t$. Then both sides are rational functions of $\alpha$. Hence formula (7.6) remains valid for general $\alpha$.

REMARK 7.3. Formula (7.6) is the explicit version of formula (7.2) for the intertwining function $\phi_{+}$; for $\phi_{-}$we obtain the same formula, because of the intrinsic symmetry of the continuous symmetric Hahn polynomials. Also the consideration of associated intertwining functions on the homogeneous space $G / M \bar{N}$ (where $\bar{N}=\theta N, \theta$ the Cartan involution on $G$ ) leads again to formula (7.6).

7.2 Associated intertwining functions on the space $G / H$. Now we consider the associated intertwining functions on the non-Riemannian symmetric space $G / H$, where $H=O_{0}(1, d-1)$. Note that $G$ admits the decomposition $G=K A H$. The subspace of left $K$ - and right $H$-invariant functions of the Casimir eigenspace containing $\phi$ has dimension two. It is spanned by an even function given explicitly by

$$
\phi_{e}\left(k \exp \left(t X_{1}\right) h\right)=\phi_{\lambda}^{\left(-\frac{1}{2}, \alpha\right)}(t) \quad(k \in K, t \in \mathbb{R}, h \in H)
$$

and an odd function given explicitly by

$$
\phi_{o}\left(k \exp \left(t X_{1}\right) h\right)=\sinh t \phi_{\lambda}^{\left(\frac{1}{2}, \alpha\right)}(t) \quad(k \in K, t \in \mathbb{R}, h \in H) .
$$


If $\lambda$ is not degenerate, it follows as in the case of $G / M N$ that $\phi_{e}$ and $\phi_{o}$ are intertwining functions on $G / H$, namely generalized matrix elements of $\pi_{\lambda}$ with distribution vectors $e_{e}$ and $e_{o}$,

$$
\phi_{e}(g)=\left\langle\pi_{\lambda}(g) e_{e}, e_{0}\right\rangle, \phi_{o}(g)=\left\langle\pi_{\lambda}(g) e_{o}, e_{0}\right\rangle \quad\left(g \in G ; e_{e}, e_{o} \in \mathcal{H}^{-\infty}(\pi)\right) .
$$

On $\exp \mathbb{R} X_{1}$, the associated intertwining functions can be expressed in terms of Jacobi functions too, namely as linear combinations of the functions

$$
(\cosh t)^{k} \phi_{\lambda}^{\left(-\frac{1}{2}, \alpha+k\right)}(t) \text { and }(\cosh t)^{k} \sinh t \phi_{\lambda}^{\left(\frac{1}{2}, \alpha+k\right)}(t)
$$

$c f$. Limić, Niederle \& Raczka [19] and Faraut [8, §V11]. The correct constants can be calculated in precisely the same way as in the case of the homogeneous space $G / K$ (see formulas (6.7)-(6.10)); note that $\phi_{e}^{k}$ is an even or an odd function on $\mathbb{R}$ according to whether $k$ is even or odd and $\phi_{o}^{k}$ is an odd or an even function on $\mathbb{R}$ according to whether $k$ is even or odd. As a result we obtain that $\phi_{e}^{k}\left(\exp t X_{1}\right)$ equals

$$
\frac{(-i)^{k}(2 \alpha)_{k}}{2^{k}(\alpha)_{k}}\left(\frac{-i \lambda}{2}+\frac{\alpha}{2}+\frac{1}{4}\right)_{k / 2}\left(\frac{-i \lambda}{2}-\frac{\alpha}{2}+\frac{1}{4}\right)_{-k / 2}(\cosh t)^{k} \phi_{\lambda}^{\left(-\frac{1}{2}, \alpha+k\right)}(t)
$$

or

(7. 10) $\frac{(-i)^{k}(2 \alpha)_{k}}{2^{k-1}(\alpha)_{k}}\left(\frac{-i \lambda}{2}+\frac{\alpha}{2}+\frac{1}{4}\right)_{(k+1) / 2}\left(\frac{-i \lambda}{2}-\frac{\alpha}{2}+\frac{1}{4}\right)_{(1-k) / 2}(\cosh t)^{k} \sinh t \phi_{\lambda}^{\left(\frac{1}{2}, \alpha+k\right)}(t)$

if $k$ is even or odd, respectively, and $\phi_{o}^{k}\left(\exp t X_{1}\right)$ equals

$$
\frac{(-i)^{k}(2 \alpha)_{k}}{2^{k}(\alpha)_{k}}\left(\frac{-i \lambda}{2}+\frac{\alpha}{2}+\frac{3}{4}\right)_{k / 2}\left(\frac{-i \lambda}{2}-\frac{\alpha}{2}+\frac{3}{4}\right)_{-k / 2}(\cosh t)^{k} \sinh t \phi_{\lambda}^{\left(\frac{1}{2}, \alpha+k\right)}(t)
$$

or

$$
\frac{(-i)^{k}(2 \alpha)_{k}}{2^{k+1}(\alpha)_{k}}\left(\frac{-i \lambda}{2}+\frac{\alpha}{2}+\frac{3}{4}\right)_{(k-1) / 2}\left(\frac{-i \lambda}{2}-\frac{\alpha}{2}+\frac{3}{4}\right)_{-(k+1) / 2}(\cosh t)^{k} \phi_{\lambda}^{\left(-\frac{1}{2}, \alpha+k\right)}(t)
$$

if $k$ is even or odd, respectively. The explicit expression for the differential operator $X_{1}$ acting from the left on right $H$-invariant functions $f$ on $G$ can be found in the same way as formula (6.17). The result is

$$
\left(X_{1} f\right)\left(k_{\theta} \exp t X_{1}\right)=\left(\cos \theta \frac{\partial}{\partial t}-\tanh t \sin \theta \frac{\partial}{\partial \theta}\right) f\left(k_{\theta} \exp t X_{1}\right) .
$$

This leads to the explicit version of formula (7.2) for associated intertwining functions on $G / H$. 
PROPOSITION 7.4. For all $\lambda \in \mathbb{C}, k \in \mathbb{Z}_{+}, \alpha=0, \frac{1}{2}, 1, \ldots$ and $\epsilon \in\{e, o\}$ we have

$$
\begin{aligned}
p_{k}\left(\frac{i}{2}\left(\cos \theta \frac{\partial}{\partial t}-\tanh t \sin \theta \frac{\partial}{\partial \theta}\right) ; \frac{\alpha}{2}+\frac{1}{4}+\frac{i \lambda}{2},\right. & \left.\frac{\alpha}{2}+\frac{1}{4}-\frac{i \lambda}{2}\right) \phi_{\epsilon}\left(\exp t X_{1}\right) \\
& =\phi_{\epsilon}^{k}\left(\exp t X_{1}\right) R_{k}^{(\alpha-1 / 2, \alpha-1 / 2)}(\cos \theta) .
\end{aligned}
$$

Here $\phi_{\epsilon}$ is given by (7.7), (7.8), $\phi_{\epsilon}^{k}$ by (7.9)-(7.12).

\section{REFERENCES}

1. R. Askey and J. Wilson, A set of hypergeometric orthogonal polynomials, SIAM J. Math. Anal. 13(1982), 651-655.

2. __ Some basic hypergeometric orthogonal polynomials that generalize Jacobi polynomials, Mem. Amer. Math. Soc. (319) 54(1985).

3. T. S. Chihara, An introduction to orthogonal polynomials, Gordon and Breach, 1978.

4. J. Dixmier and P. Malliavin, Factorisations de fonctions et de vecteurs indefiniment différentiables, Bull. Sci. Math. (2) 102(1978), 307-330.

5. A. Erdélyi, Zur Theorie der Kugelwellen, Physica 4(1937), 107-120.

6. A. Erdélyi, W. Magnus, F. Oberhettinger and F. G. Tricomi, Higher transcendental functions, Vol. I, McGraw-Hill, 1953.

7. _ـ Higher transcendental functions, Vol. II, McGraw-Hill, 1953.

8. J. Faraut, Distributions sphériques sur les espaces hyperboliques, J. Math. Pures Appl. (9) 58(1979), 369444

9. __ Analyse harmonique sur les paires de Guelfand et les espaces hyperboliques, in Analyse harmonique, CIMPA, Nice, 1982, 315-446.

10. __ Analyse harmonique et fonctions spéciales, in Deux cours d'analyse harmonique, Birkhaüser, Boston, 1987, 1-151.

11. M. Flensted-Jensen, Non-Riemannian symmetric spaces, CBMS Regional Conference Series 61, American Mathematical Society, 1986.

12. I. M. Gelfand and G. E. Shilov, Generalized functions, I. Properties and operations, Academic Press, 1964.

13. S. Helgason, A duality for symmetric spaces with applications to group representations, II. Differential equations and eigenspace representations, Adv. in Math. 22(1976), 187-219.

14. $\longrightarrow$ Groups and geometric analysis, Academic Press, 1984.

15. S. Karlin and J. L. McGregor, The Hahn polynomials, formulas and an application, Scripta Math. 26(1961), $33-46$.

16. T. H. Koornwinder, The representation theory of $\mathrm{SL}(2, \mathbb{R})$, a global approach, Report ZW 145/80, Mathematisch Centrum, Amsterdam, 1980.

17. J__ Jacobifunctions and analysis on noncompact semisimple Lie groups, In: Special functions: Group theoretical aspects and applications (ed. R. A. Askey, T. H. Koornwinder and W. Schempp), Reidel, 1984, $1-85$.

18. Group theoretic interpretations of Askey's scheme of hypergeometric orthogonal polynomials, in Orthogonal polynomials and their applications, (ed. M. Alfaro, J. S. Dehesa, F. J. Marcellan, J. L. Rubio de Francia and J. Vinuesa), Lecture Notes in Math. 1329, Springer, 1988, 46-72.

19. N. Limić, J. Niederle and R. Raczka, Continuous degenerate representations of noncompact rotation groups. II, J. Math. Phys. 7(1966), 2026-2035.

20. B. van der Pol, A generalization of Maxwell's definition of solid harmonics to waves in $n$ dimensions, Physica 3(1936), 393-397.

21. A. Strasburger, Inducing spherical representations of semi-simple Lie groups, Dissertationes Math. (Rozprawy Mat.) 122(1975).

22. G. Szegö, Orthogonal polynomials, Amer. Math. Soc. Colloquium Publications 23, American Mathematical Society, Fourth edition, 1975.

23. N. Ya. Vilenkin, Special functions and the theory of group representations, Translations of Mathematical Monographs 22, American Mathematical Society, 1968. 
24. N. R. Wallach, Real reductive groups I, Academic Press, 1988.

25. G. N. Watson, A treatise on the theory of Bessel functions, Cambridge University Press, Second edition, 1944.

Mathematisches Institut

Sidlerstrasse 5

CH-3012 Bern

Switzerland

email:badertscher@mai.unibe.ch

$C W I$

P.O. Box 4079

1009 AB Amsterdam

The Netherlands

Present address of second author:

University of Amsterdam

Faculty of Mathematics and Computer Science

Plantage Muidergracht 24

1018 TV Amsterdam

The Netherlands

email: thk@fwi.uva.nl 